\title{
PUNTOS DE ALIMENTACIÓN SALUDABLE - PAS, TRANSFORMACIÓN DEL AMBIENTE CONSTRUIDO PARA DISMINUIR LOS IMPACTOS DEL SOBREPESO Y OBESIDAD EN LA POBLACIÓN DE CALI
}

\section{Healthy food center - hfc, transforming the built environment to reduce the impact of overweight and obesity among the population of Cali}

\author{
DANIEL CUARTAS 1 \\ 1Universidad del Valle.
}

E-mail: decuartas@grupogesp.org

\section{Resumen}

La alta prevalencia del sobrepeso y obesidad convierten a esta enfermedad en una de las principales preocupaciones a nivel mundial, sus características hacen que se relacione, afecte y agudice otras enfermedades. Estudios plantean que el sobrepeso y la obesidad no son una enfermedad individual y que por el contrario es una enfermedad social, relacionada con los ambientes construidos. Este trabajo busca describir la metodología para localización de Puntos de Alimentación Saludable - PAS, desde los cuales se distribuya y fomente comida saludable como estrategia para transformar los ambientes construidos y disminuir los impactos del sobrepeso y la obesidad en la población de la ciudad de Cali.

Palabras claves: Sobrepeso, Obesidad, Ambiente construido, Comida saludable, Análisis exploratorio de datos espaciales.

\begin{abstract}
The high prevalence of overweight and obesity make this disease a major concern worldwide, its features make it relates, affects and exacerbates other diseases. Studies suggest that overweight and obesity are not an individual disease and instead is a social disease, related to the built environment. This work describe the methodology for locating Healthy Food Center - HFC, from which distributes and promotes healthy food as a strategy to transform the built environment and reduce the impacts of overweight and obesity in the population of the city of Cali.
\end{abstract}

Keywords: Overweight, Obesity, Built environment, Healthy food, Spatial data analysis - ESDA 


\section{INTRODUCCIÓN}

La alta prevalencia del sobrepeso y obesidad han convertido a esta enfermedad en una de la principales preocupaciones a nivel mundial (Formiguera \& Canon 2004), la Organización Mundial de la Salud - OMS - ha informado que para el año 2015 una importante cantidad de personas (2.300 millones con sobrepeso y 700 millones de obesos) presentaran este evento en salud (OMS).

De igual manera el sobrepeso y la obesidad se constituyen en un factor de riesgo para otros eventos en salud como problemas del corazón, diabetes tipo 2, apnea de sueño, osteoartritis y asma. También aumenta el riesgo de morbilidad y mortalidad de cáncer (Nejat et al. 2010). El Centro para el control y prevención de enfermedades (CDC) estimo en el año 2000 que 15.2\% (365000) de la muertes de estados unidos estuvieron asociadas a una mala dieta y a inactividad física (ICBF 2005). De acuerdo a cifras de la Encuesta Nacional de la Situación Nutricional en Colombia, 2005 - ENSIN Colombia y en especial Cali, presentan altos niveles de sobrepeso y obesidad.

Desde diferentes perspectivas se plantea que el sobrepeso y la obesidad no solo son una enfermedad del ámbito individual y que por el contrario es una enfermedad social en la que participan diferentes elementos en los que se incluyen los ambientes construidos. Sin embargo también es importante tener en cuenta los cambios culturales resultantes de la modernidad occidental, la necesidad de incrementar el consumo (hasta un consumismo) como base del sistema económico ha llevado a una producción intensiva sin límite en el uso de los recursos naturales.

Dicho así, la relación entre la configuración actual de los ambientes construidos, el sobrepeso y la obesidad, es compleja, y expresa los cambios culturales recientes. En este marco este trabajo se propone identificar la distribución espacial del sobrepeso y la obesidad a partir de los datos de la ENSIN 2010. Posteriormente seleccionará áreas de acuerdo a la prevalencia de sobrepeso y obesidad, condiciones socioeconómicas y resultados de la ENSIN que requieran de la localización y desarrollo de Puntos de Alimentación Saludable - PAS, desde los cuales se distribuya y fomente comida saludable como estrategia para disminuir los impactos del sobrepeso y la obesidad en la población de la ciudad de Cali.

\section{EL SOBREPESO Y LA OBESIDAD UNA TENDENCIA A NIVEL GLOBAL Y LOCAL}

Una de las principales preocupaciones a nivel mundial en materia de salud pública es la alta prevalencia de obesidad y sobrepeso, como resultado de estilos de vida poco saludables (Formiguera \& Canon 2004). Según datos de la Organización Mundial de la Salud para el año de 2005 alrededor del mundo 1.600 millones de personas tenían sobrepeso y cerca de 400 millones eran obesos, esta misma institución estima que para el 2015, las personas con sobrepeso serán 2.300 millones y los obesos ascenderán a 700 millones (OMS).

Adicionalmente está claramente identificado la relación del sobrepeso y la obesidad con otras enfermedades hipertensivas, cerebrovasculares e isquémicas (Nejat et al. 2010), y en general con el grupo de enfermedades denominadas como enfermedades crónicas no transmisibles. En Colombia se ha desarrollado en dos ocasiones la Encuesta Nacional de la Situación Nutricional -ENSIN- (2005, 2010), pero solo se conocen los datos completos de la ENSIN 2005, de la ENSIN 2010 se ha publicada un resumen ejecutivo que permite establecer que el sobrepeso y la obesidad ha aumentado en Colombia, paso del $45.9 \%$ en el 2005 al 51.2\% en el 2010, (Figura 1), lo que demuestra que Colombia no es la excepción en la tendencia mundial (ICBF 2005, 2011).
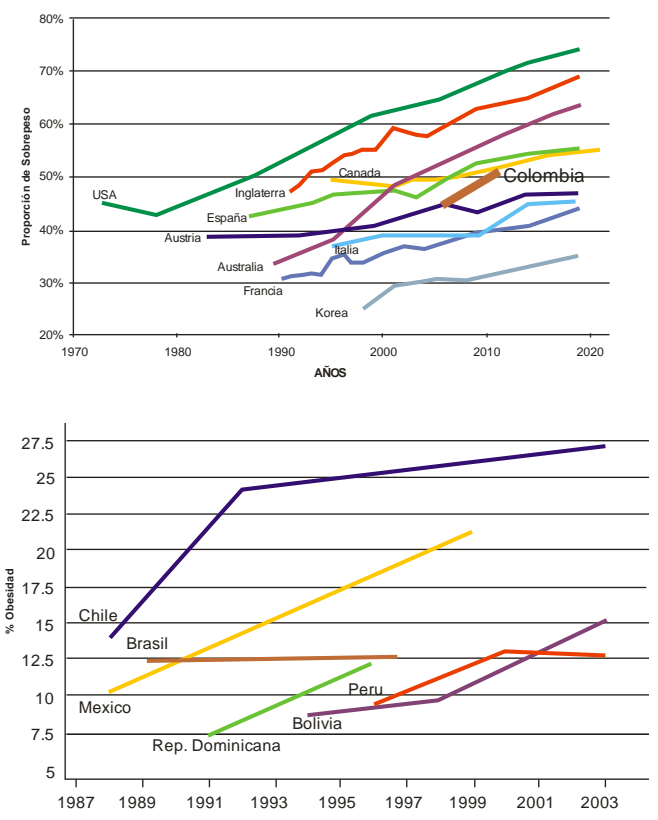

Figura 1. Tendencia de sobrepeso y obesidad en diferentes países. Adaptado de Selassie \& Sinha (2011), ICBF (2011). 
De acuerdo con los resultados reportados en la ENSIN (2005), la ciudad de Cali posee la prevalencia de obesidad y sobrepeso se encuentra por encima del promedio nacional. Para el grupo compuestos por personas entre los 10 y los 17 años el promedio nacional de sobrepeso es del 10,3\% y en la ciudad de Cali se presenta en el mismo grupo etáreo una prevalencia del 16,3\%. De igual manera en el grupo de los 18 a los 64 años, el promedio nacional fue del $46 \%$ y la prevalencia para Cali fue del 50,9\%.

Adicionalmente, al observar la cantidad de tiempo que los niños entre los 5 y los 12 años dedican a ver televisión encontraron que a nivel nacional el $56,3 \%$ dedica dos horas o más y que el $21,9 \%$ dedica 4 horas o más, para Cali la proporción de personas en el mismo grupo de edad correspondió al 70,5\% y al 28,8\% respectivamente.

De manera consecuente se identificó que Cali es una de las regiones con mayor proporción de personas inactivas en el grupo de edad de los 18 a los 64 años el 70,8\% son personas inactivas mientras que el promedio nacional fue del 53,4\% (ICBF 2005).

La tendencia a nivel global ha motivado el desarrollo de un considerable número de estudios (Fabricatore \& Thomas 2006, Glanz et al. 2005, Glanz et al. 2007, Saelens et al. 2007), (en Colombia apenas se empieza a desarrollar algunos estudios) en los cuales plantean que la obesidad no corresponde a un asunto meramente individual y se viene estableciendo una relación entre los niveles de obesidad de la población y las características de los ambientes construidos en los que las comunidades desarrollan la vida.

Estos estudios abordan los ambientes construidos como facilitadores del acceso a alimentos que favorecen la ganancia de peso o que no propician que las personas realicen algún tipo de actividad física. Observan el acceso a la comida, los tipos de comida que se ofrecen, el costo de la misma, los patrones culturales y los patrones de alimentación y en algunos, las particularidades de los lugares donde se compra la comida.

Al observar lo que algunos estudios vienen planteando sobre la necesidad de abordar el problema de la obesidad no como un asunto individual si no como una enfermedad en la que los ambientes construidos tienen relación, resulta conveniente revisar cual ha sido la construcción y configuración de dichos ambientes en la ciudad de Cali y como en estos no existe ninguna infraestructura que facilite la adquisición de comida saludable, especialmente en grupos poblacionales de bajos niveles económicos.

\section{EL PROCESO DE URBANIZACIÓN DE COLOMBIA Y CALI}

En Colombia se puede detectar un patrón de localización poblacional diferente a partir de 1936 como resultado de una serie de factores sociales, políticos y económicos, la agudización del conflicto interno, la tenencia de la tierra y el proceso de industrialización, propiciaron que cierta población abandonará el campo para localizarse en núcleos concentrados, dando lugar al proceso de urbanización del país (Zambrano \& Bernard 1993, Escobar).

En el censo que se realizó en 1951 el cambio de vocación de un país rural a urbano fue mucho más notorio, mientras que para el censo de 1938 las población localizada en núcleos urbanos correspondía al 29\% para el censo de 1951 era del 42,6\% y para el censo de 2005 la concentración urbana alcanzo el 75,9\% (Zambrano \& Bernard 1993, DANE 2005).

En este sentido, la ciudad de Cali ha sufrido grandes transformaciones en los últimos 60 años, ha experimentado un marcado cambio en la estructura poblacional, ha sido una ciudad receptora de población que se desplazó de otros lugares del país, especialmente de la costa del pacifico y del sur, además ha presentado un proceso de urbanización acelerado y poco planificado. La concentración en la cabecera municipal de Cali se desarrolló más rápidamente que en Colombia, para 1951 en el área urbana se localizaba el 85\% de la población del municipio y a partir de 1973 la concentración urbana corresponde al 98\%. La concentración de población en la ciudad de Cali, produjo una gran demanda vivienda que no era cubierta, lo que desencadeno el desarrollo de asentamientos subnormales especialmente en el oriente y nororiente de la ciudad, así como en la ladera. Este proceso de principios de la década de 1980 que continúo incluso durante los últimos años de manera menos intensa, ha provocado una expansión no planificada de la ciudad (Zambrano \& Bernard 1993).

En la actualidad existe un contexto que reclama de intervenciones en los ambientes construidos para enfrentar la 
tendencia creciente de sobrepeso y obesidad en la ciudad de Cali:

- Alta prevalencia de sobrepeso en la ciudad de Cali por encima del promedio nacional.

-Una alta proporción de la población es físicamente inactiva.

-Expansión urbana poco planificada, concentración de población en algunos sectores, construcción y configuración de ambientes urbanos que afectan las formas como se materializa el acto de vivir.

Sin embargo, una mirada exclusiva a las características de los ambientes construidos resulta insuficiente para comprender la influencia de estos en el sobrepeso y la obesidad, es necesario identificar patrones culturales característicos de la actual sociedad caleña de tal forma que sea posible localizar PAS como un servicio básico de la comunidad.

\section{DESARROLLO, ESTILOS DE VIDA, AMBIENTES COSTRUIDOS Y OBESIDAD}

Así como se mencionó que en Colombia los más de 50 años de conflicto interno han modificado los ambientes construidos en los núcleos urbanos y ciudades, resulta impensable no establecer un vínculo entre sobrepeso, obesidad, ambientes construidos y desarrollo y modernidad.

La división del mundo entre países desarrollados y subdesarrollados tuvo su origen a partir del discurso de posesión del presidente de los Estados Unidos Harry Truman en 1949, esta división estuvo acompañada de la implementación de programas para el desarrollo de los países subdesarrollados, basados en el aumento de la producción, sustentada en la aplicación del conocimiento técnico y científico (Escobar 2010). Estos programas de desarrollo del tercer mundo también implicaron la transformación cultural de las sociedades, debía darse la transición de sociedades tradicionales a sociedades modernas, Arturo Escobar lo describe de la siguiente manera:

"Primero, el hecho de que el desarrollo es un proyecto tanto económico (capitalista e imperial) como cultural. Es cultural en dos sentidos: surge de la experiencia particular de la modernidad europea; y subordina a las demás culturas y conocimientos, las cuales pretende transformar bajo principios occidentales. Segundo, el desarrollo y la modernidad involucran una serie de principios: el individuo racional, no atado ni a lugar ni a comunidad; la separación de naturaleza y cultura; la economía separada de lo social y lo natural; la primacía del conocimiento experto por encima de todo otro saber" (Escobar 2010). Esta transformación cultural produjo cambios en los estilos de vida, los centros urbanos adquirieron gran importancia en la relación centro - periferia, el aumento de la producción apoyada en el conocimiento tecnológico y científico, produjo un cambio en la oferta de alimentos y comidas, cada vez más se consumen productos preparados y elaborados, ricos en azucares refinados y grasas trans. Se disminuyó los niveles de actividad física al tener ciudades expandidas en las cuales deben realizarse grandes recorridos en transporte motorizado, se fomentó el uso del automóvil y adicionalmente el consumo de televisión, videojuegos e Internet se convirtió en uno de las principales actividades de tiempo libre que realizan los jóvenes.

Los anteriores elementos característicos del mundo moderno y desarrollado, también hacen parte del mundo subdesarrollado e influyen al configurar condiciones y características de los ambientes construidos, así como factores relacionados con los niveles de sobrepeso y obesidad a nivel mundial, nacional y por supuesto de la ciudad de Cali.

Alternativas a las ideas del mundo desarrollado son posibles, pero se requiere de volver la mirada a las culturas locales, pueden identificarse características que permitan encontrar una forma distinta de concebir los ambientes construidos y articularlos con elementos propios del tema de interés como son los hábitos alimenticios, la actividad física y los estilos de vida, además se requiere cambios en el ambiente construido, diseño de infraestructura que favorezca el acceso a alimentos saludables.

\section{LOCALIZACIÓN DE PUNTOS DE ALIMENTACIÓN SALUDABLE - PAS, HACIA LA RECONFIGURACIÓN DEL AMBIENTE CONSTRUIDO}

Teniendo en cuenta que la prevalencia de sobrepeso y obesidad en Cali es más alta que el promedio nacional y que sigue tendencias generales, es necesario el desarrollo de intervenciones a distintos niveles para con el objetivo de revertir la tendencia y mejorar la calidad de vida de la población de la ciudad. En este sentido el presente trabajo 
busca definir la metodología para la identificación de áreas prioritarias en la ciudad de Cali debido a los altos niveles de sobrepeso y obesidad que allí se presentan, áreas susceptibles a la localización de PAS que permitirán el acceso a alimentación saludable de la población.

La fuente de información inicial se constituye la ENSIN desarrollada en el 2005 y en el 2010, las cuales utilizan como fuente cartográfica base los segmentos urbanos definidos por el Departamento Administrativo Nacional de Estadistica DANE. A partir de las fuentes de datos de ENSIN y de la fuente Cartográfica del DANE se realiza inicialmente un análisis exploratorio de datos espaciales - AEDE con los datos de sobrepeso y obesidad.

El AEDE es un método de estadística descriptiva espacial que permite describir y visualizar la distribución de los datos de sobrepeso y obesidad. De tal forma que en el caso de existir, se puedan identificar localizaciones atípicas, esquemas de asociación espacial y estructuras espaciales de dicha distribución (Barcellos 2003, Coro 2006). Con el AEDE es posible identificar tres elementos en la variable geográfica de estudio, la tendencia, la asociación y/o dependencia espacial y la concentración o atípicos espaciales (Coro 2006, Ministério da Saúde do Brasil \& Fundação Oswaldo Cruz 2006). Estos elementos serán analizados para dos variables que miden la ocurrencia de casos, la primera es el número de casos y la segunda es la tasa de ocurrencia.

El AEDE que se propone desarrollar para las dos variables mencionadas es el siguiente:

1. Análisis de densidad de casos por año y para todo el periodo (Ministério da Saúde do Brasil \& Fundação Oswaldo Cruz 2007a).

2. Análisis de tendencia espacial por año y para todo el periodo del número de casos y de la tasa por comuna (Coro 2006, Ministério da Saúde do Brasil \& Fundação Oswaldo Cruz 2007b).

3. Análisis de asociación espacial por año y para todo el periodo del número de casos y de la tasa por comuna (Coro 2006, Ministério da Saúde do Brasil \& Fundação Oswaldo Cruz 2007b).

4. Análisis de concentración espacial por año y para todo el periodo del número de casos y de la tasa por comuna (Coro
2006, Ministério da Saúde do Brasil \& Fundação Oswaldo Cruz 2007b).

5. Análisis de cluster (Ministério da Saúde do Brasil \& Fundação Oswaldo Cruz 2007b).

Con la información que suministre el anterior análisis complementada con características socioeconómicas e información de hábitos alimenticios y de actividad física tomada del ENSIN 2010, se identificaran áreas prioritarias en la ciudad que son susceptibles para la localización de PAS.

Los PAS son comedores comunitarios que ofrecen una alimentación balanceada de acuerdo con criterios nutricionales y características locales. Las comidas ofrecidas serán de bajo costo pero de excelente calidad, facilitando el acceso de las personas del sector a una alimentación saludable. De igual manera los PAS desarrollaran actividades de educación comunitaria para promocionar estilos de alimentación saludable, enseñar preparaciones nutritivas, gustosas, con los alimentos que disponen las comunidades y de poco costo económico.

Es importante que los PAS se encuentren rodeados de espacios públicos que faciliten la realización de actividad física, además que permitan el encuentro de la comunidad, que generen procesos de construcción social alrededor del comedor comunitario. Por último alrededor de los PAS se deben convocar tanto a instituciones públicas como privadas, es necesario que los supermercados, las EPS, el gobierno municipal, departamental y nacional participen en estos proyectos, debido a que revertir la tendencia de la obesidad requiere un esfuerzo de todos, además que los beneficios al tener menos factores de riesgo de ocurrencia de enfermedades crónicas no transmisibles significan la disminución de los costos en salud que al final son socializados.

\section{CONCLUSIONES}

La tendencia al incremento del sobrepeso y la obesidad tiene implicaciones tanto a nivel global como local, esta se ha aumentado de forma significativa en los últimos 30 años y se ha convertido en un problema de salud pública y en uno de los principales temas en las agendas de organizaciones internacionales. 
El sobrepeso y la obesidad no es un asunto individual, es un problema social en el que intervienen factores culturales, biológicos, sociales, económicos, contextuales (ambiente construido) y características del modelo de desarrollo actual, que busca aumentar el consumo, fundamentado en una cultura de poseer cosas que se usan y se tiran.

Adicionalmente la manera como se ha desarrollado los procesos de urbanización en Colombia también ha generado ambientes construidos que facilitan el acceso a ciertos tipos de alimentos e inhiben la realización de actividad física de las personas.

Bajo estas circunstancias es necesario que desde iniciativas colectivas entre instituciones públicas, privadas y organizaciones no gubernamentales se desarrollen iniciativas que busquen revertir la tendencia de incremento del sobrepeso y obesidad en la ciudad y en Colombia. Los PAS se constituyen en una excelente oportunidad de intervención física y social de comunidades que presentan altos niveles de sobrepeso y obesidad en la búsqueda de facilitar el acceso y configurar comunidades más equitativas.

\section{REFERENCIAS}

BARCELLOS, C. 2003. Unidades y Escalas en Los Análisis Espaciales en Salud. Revista Cubana de Salud Pública 29(4): 307-313.

CORO, C. 2006. Análisis estadístico de datos geográficos en geomarketing : el programa GeoDa. Revista Distribución y Consumo. Universidad Autonoma de Madrid. Mar - Abril

DANE. 2005. Boletin Censo General 2005 http://www.dane.gov.co/files/censo2005/PERFIL_PDF_CG 2005/00000T7T000.PDF

ESCOBAR, A. 2010. Una minga para el postdesarrollo: lugar, medio ambiente y movimientos sociales en las transformaciones globales. Universidad Nacional Mayor de San Marcos, Lima. 222 pp.

ESCOBAR, G. La población en Santiago de Cali. Cali: disponible:http:// planeacion.cali.gov.co/Publicaciones/Dem ografia/La\%20poblacion\%20en\%20Cali.pdf
FABRICATORE, A. \& THOMAS, W. 2006. Obesity. Annual Review of Clinical Psychology 2: 357-377.

FORMIGUERA, X. \& CANON, A. 2004. Obesity: epidemiology and clinical aspects. Best Practice \& Research Clinical Gastroenterology 18(6): 1125-1146.

GLANZ, K. SALLIS, J. F. SAELENS, B. E. \& FRANK, L. D. 2005. Healthy nutrition environments: Concepts and measures. American Journal of Health Promotion 19(5): 330333

GLANZ, K. SALLIS, J. F. SAELENS, B. E. \& FRANK, L. D. 2007. Nutrition environment measures study in stores (NEMS-S): Development and Evaluation. American Journal of Preventive Medicine 32: 282-289.

ICBF, INSTITUTO COLOMBIANO DE BIENESTAR FAMILIA. 2005. Encuesta nacional de la situación nutricional. Imprenta Nacional de Colombia, Bogotá. 445 pp.

ICBF, INSTITUTO COLOMBIANO DE BIENESTAR FAMILIA. 2011. Resumen Ejecutivo Encuesta nacional de la situación nutricional, 2010. Bogotá: Imprenta Nacional de Colombia.

MINISTÉRIO DA SAÚDE DO BRASIL \& FUNDAÇÃO OSWALDO CRUZ. 2006. Abordagens Espaciais na Saúde Pública. Capacitaçao e Actualizaçao em Geoprocessamento em Saúde. Série B: Textos Básicos de Saúde, Brasilia. Vol 1.

MINISTÉRIO DA SAÚDE DO BRASIL \& FUNDAÇÃO OSWALDO CRUZ. 2007a. Sistemas de Informaçoes Geográficas e Análise Espacial na Saúde Pública. Capacitaçao e Actualizaçao em Geoprocessamento em Saúde. Série B: Textos Básicos de Saúde, Brasilia. Vol 2.

MINISTÉRIO DA SAÚDE DO BRASIL \& FUNDAÇÃO OSWALDO CRUZ. 2007b. Introduçao à Estadística Espacial para a Saúde Pública. Série: Capacitaçao e Actualizaçao em Geoprocessamento em Saúde. Série B: Textos Básicos de Saúde, Brasilia. Vol 3.

NEJAT, E. J. POLOTSKY, A. J. \& PAL, L. 2010. Predictors of chronic disease at midlife and beyond - the Elath risks of obesity. Maturitas 65: 106-111.

OMS, ORGANIZACIÓN MUNDIAL DE LA SALUD. Obesidad y Sobrepeso. Nota descriptiva No. 311 Disponible: 
Ambiente y Sostenibilidad 2011 (1): 32-38

Revista del Doctorado Interinstitucional en Ciencias Ambientales

ISSN: 2339-3122

http://www.who.int/mediacentre/factsheets/fs311/es/inde x.htmlfuente

SAELENS, B. E. GLANZ, K. SALLIS, J. F. \& FRANK, L. D. 2007. Nutrition environment measures study in restaurants (NEMS-R): Development and Evaluation. American Journal of Preventive Medicine 32: 273-281.

ZAMBRANO, F. \& BERNARD, O. 1993.Ciudad y territorio, el proceso de doblamiento en Colombia. Bogotá: Tercer mundo editores. $297 \mathrm{pp}$. 